\title{
Cahiers de Narratologie
}

\section{Enquête sur le graffiti en Chine}

Shiyan Li

\section{OpenEdition}

\section{Journals}

Electronic version

URL: http://journals.openedition.org/narratologie/7400

DOI: 10.4000/narratologie. 7400

ISSN: 1765-307X

\section{Publisher}

LIRCES

\section{Electronic reference}

Shiyan Li, «Enquête sur le graffiti en Chine », Cahiers de Narratologie [Online], 29 | 2015, Online since 05 February 2016, connection on 01 May 2019. URL : http://journals.openedition.org/narratologie/7400 ; DOI : $10.4000 /$ narratologie. 7400

This text was automatically generated on 1 May 2019.

Article L.111-1 du Code de la propriété intellectuelle. 


\title{
Enquête sur le graffiti en Chine
}

\author{
Shiyan Li
}

\section{L'état des lieux de la recherche actuelle sur l'art du graffiti en Chine}

1 En Chine l'art du graffiti est introduit dans les années 1980 à travers certaines revues artistiques, mais c'est en 1990 que cet art est apparu pour la première fois sur la scène artistique grâce à l'artiste Zhang Dali (né en 1963 à Heilongjiang). Ce dernier est considéré comme le premier artiste à proposer un art du graffiti par les critiques d'art influents.

Actuellement en Chine, il y a très peu de recherches universitaires consacrées à l'art du graffiti. À ma connaissance, il existe deux mémoires de master II: l'un est celui de Wang Jing, intitulé L'expression de l'anti-culture dans l'art du graffiti aujourd'hui (Fanwenhuaxing zai dangdai tuyayishuzhong de biaoxian) soutenu en 2007 au Hubei Institute of Fine Arts, l'autre est celui de Wei Hua : La recherche de faisabilité du développement de l'art du graffiti en Chine ( Tuyayishu zai zhongguo fazhan de kexingxing yanjiu), soutenu en 2008 à la Central China Normal University. Aucun historien d'art ou critique d'art chinois de premier plan ne s'intéresse au développement du graffiti en Chine. C'est souvent à travers les différents médias et surtout par les messages de bloggeurs passionnés de graffiti que le public peut recevoir des informations dispersées sur cette forme d'expression nouvelle désormais considérée comme relevant de l'art en Chine. En Occident on compte quelques études consacrées au graffiti en Chine: celle de Minna Valjakka, chercheuse à l'Université d'Helsinki qui publie en 2011 un article intitulé "Graffiti in China - Chinese Graffiti' ${ }^{1}$ ? ; celle de Norbert Kirbach, artiste allemand et spécialiste du graffiti qui publie un article intitulé «Street art in Hong Kong - Communication in Public Spaces » en 2014 dans l'ouvrage collectif intitulé Crossing China. Land of Rising Art Scene ${ }^{2}$; celle de Pan Lü, chercheuse à l'Harvard-Yenching Institute qui publie en 2015 un livre intitulé Aesthetizing Public Space Street, Street Visual Politics in East Asian Cities ${ }^{3}$ dans lequel il y a deux parties consacrées au sujet qui nous intéresse. Des documentaires viennent compléter ce corpus de publications pour aider à connaître ce champ aujourd'hui à peine abordé. On notera 
celui de l'artiste Seth (Julien Mallant) qui a été réalisé en 2010 et diffusé par la chaine Canal+. On trouve dans ce documentaire qui dure 52mn le premier graffeur Zhang Dali ainsi que les graffeurs issus de la génération née après 1980. Un autre documentaire important réalisé par Lance Crayon intitulé Spray Paint Beijing, Graffiti in the Capital of China (2012), très peu vu par le public chinois, a fait pas mal de bruit dans certains médias internationaux comme la BBC, The Economist, ou Time Out Beijing.

\section{La traduction du " graffiti » en chinois et la difficulté de la définition}

3 Lorsque l'art du graffiti est introduit en Chine, on utilise le terme tuya yishu pour désigner cette nouvelle forme d'art qui est née en Occident. Le binôme tuya est composé de deux sinogrammes $t u$ (涂) et $y a$ (鸦). Tu veut dire griffonner, barbouiller et $Y a$ signifie le corbeau. Ensemble, les deux sinogrammes expriment littéralement une écriture du pinceau à l'encre de Chine illisible qui ressemble à une réunion de corbeaux dont on ne peut distinguer l'un de l'autre. L'origine de ce binôme se trouve d'abord chez le poète $\mathrm{Lu}$ Tong (795-835) qui l'emploie pour désigner le griffonnage de son enfant sur la table de son père. Jusqu'aujourd'hui, on emploie encore ce mot pour désigner le dessin, la peinture et l'écriture des enfants. Un autre sens de ce mot provient du poète Li Yu (1610-1680) qui l'utilise pour décrire son écriture rapide réalisée à son gré. En même temps le mot manifeste aussi une certaine modestie de la part de l'auteur envers sa propre œuvre. Ces deux sens peuvent faire écho à l'origine du mot graffiti de l'Occident : graphein qui signifie à la fois écrire, dessiner ou peindre. Il n'est donc pas inopportun d'employer ce mot pour traduire le terme graffiti. Lorsqu'il est associé à des mots comme " art », " peinture » ou bien "street art », il renvoie à l'art du graffiti de l'Occident. Cependant, cette nouvelle forme de l'art nous rappelle aussi une pratique courante chez les lettrés traditionnels, qui jadis calligraphiaient leurs poèmes sur la surface d'un rocher, dédiée uniquement à cet usage pour qu'ils puissent s'exprimer. On peut nommer ce genre d'inscription le graffiti du lettré (wenren tuya). De telles inscriptions peuvent être ensuite gravées sur la pierre par des artisans pour y demeurer. Lorsque cette culture du lettré évolue vers l'époque contemporaine elle devient une pratique courante des masses qui fait que des visiteurs, incapables d'écrire un poème, laissent simplement une phrase, par exemple cette simple formule «Untel est passé ici » sur des monuments historiques pour indiquer la trace de leur visite ${ }^{4}$. Ce genre d'inscription, soit gravée, soit écrite, est considéré comme le graffiti inculte (buwenming tuya) dû à un comportement jugé vulgaire. C'est dans ce contexte culturel complexe, d'un côté hérité de la culture élitiste traditionnelle, de l'autre côté imprégné de la culture de masse que le tuya (graffiti) chinois va rencontrer le "graffiti moderne » de l'Occident, concept proposé par Jack Stewart pour étudier cette nouvelle forme de l'art qui se développe à partir des années 1960 dans les rues de la ville de Philadelphie aux Etats-Unis, puis à New York dans les années 1970 et $1980^{5}$. Et jusqu'aujourd'hui, les opinions sur cette nouvelle forme d'art restent très partagées en Chine. Les graffeurs font souvent référence au tuya du lettré traditionnel pour affirmer que c'est de l'art, tandis que d'autres personnes, notamment les habitants des quartiers des grandes villes, qui refusent de voir ce qu'ils considèrent comme un vandalisme de leur environnement, jugent que le graffiti est le fruit d'un comportement inculte. D'ailleurs, à leurs yeux, il est souvent synonyme de la maladie du psoriasis (Niupixian). Car le fait de peindre ou écrire sur un mur ressemble à des petites 
annonces publicitaires non officielles, mais très populaires, qui offrent des services, jugées illégales, par exemple pour soigner la maladie du psoriasis ou bien se charger de réaliser des papiers officiels, etc. C'est ainsi qu'en Chine, la réception de l'art du graffiti n'est pas à mettre sur le même plan que celle des autres formes de l'art provenant de l'Occident qui, elles, s'adressent à une certaine élite : artistes, critiques, historiens de l'art.

4 Avec ses origines en partie populaire et sa diffusion spécifique visant n'importe quel public, l'art du graffiti en Chine se développe d'une manière bien particulière. Il est d'abord reconnu par des critiques d'arts influents alertés par les œuvres de l'artiste Zhang Dali, ensuite bien vite ignoré par la critique qui laisse de côté les graffitis des générations nées après 1980, fortement influencées par la culture du Hip-hop. Enfin cette nouvelle forme d'art avec sa diffusion massive devient un outil pour la propagande du gouvernement chinois. Dans le premier temps de cet article je décrirai cette trajectoire historique et dans un deuxième temps, j'évoquerai quelques réalisations qui ont pu échapper à la surveillance du gouvernement et ont suscité ainsi des tensions politiques.

\section{La naissance de l'art du graffiti en Chine}

La naissance de l'art du graffiti est reliée directement au développement économique en Chine. Dans les années 1990, la Chine a subi de grands changements économiques et sociaux. Les grandes villes comme Pékin et Shanghai sont des chantiers immenses voués à la destruction et la construction. Des milliers de maisons anciennes sont détruites pour construire des hôtels de luxe, des bureaux, des centres commerciaux, des résidences haut de gamme sous forme de gratte-ciel. Partout on voit des grues, des échafaudages, des bulldozers, des murs brisés, des vieux quartiers en ruine. Partout on voit des sinogrammes en grandes lettres : « démolir » (拆), « délocaliser »(般迁).

6 C'est dans ce contexte, en plein désordre, que naît le premier graffiti au sens occidental. L'auteur de cette réalisation est l'artiste Zhang Dali. En 1983 il poursuit ses études à la Central Academy of Art and Design ${ }^{6}$ à Pékin. Après avoir obtenu son diplôme en 1987, il s'installe à Pékin en tant que peintre et ensuite il part pour l'Italie en 1989.

Lorsque l'artiste arrive à Bologne, il se trouve isolé dans une culture étrangère qui ne parvient pas à lui ouvrir la porte de l'histoire de l'art occidentale avec ses musées prestigieux. En 1992, par hasard, il découvre des graffitis au mur des petites ruelles de la ville. Et tout de suite, il pense que c'est un nouveau moyen de s'exprimer. Or, déjà en Chine, à travers certaines revues artistiques, il avait pris connaissance du graffiti de l'Occident mais cela n'avait pas retenu son attention à cause de sa situation stable à l'époque. Maintenant, à Bologne, le graffiti sur le mur est comme un révélateur qui lui permet de s'exprimer dans un monde inconnu. Et il commence dès lors à dessiner le contour d'une tête nue reprenant la forme de sa propre tête sur les murs de la cité italienne pour engager un dialogue avec la ville et les autres graffeurs occidentaux. Parfois la tête nue suscite des malentendus. Un exemple peut être cité ici : un jour sur la tête nue sont ajoutés la faucille et le marteau, symboles graphiques communistes. 


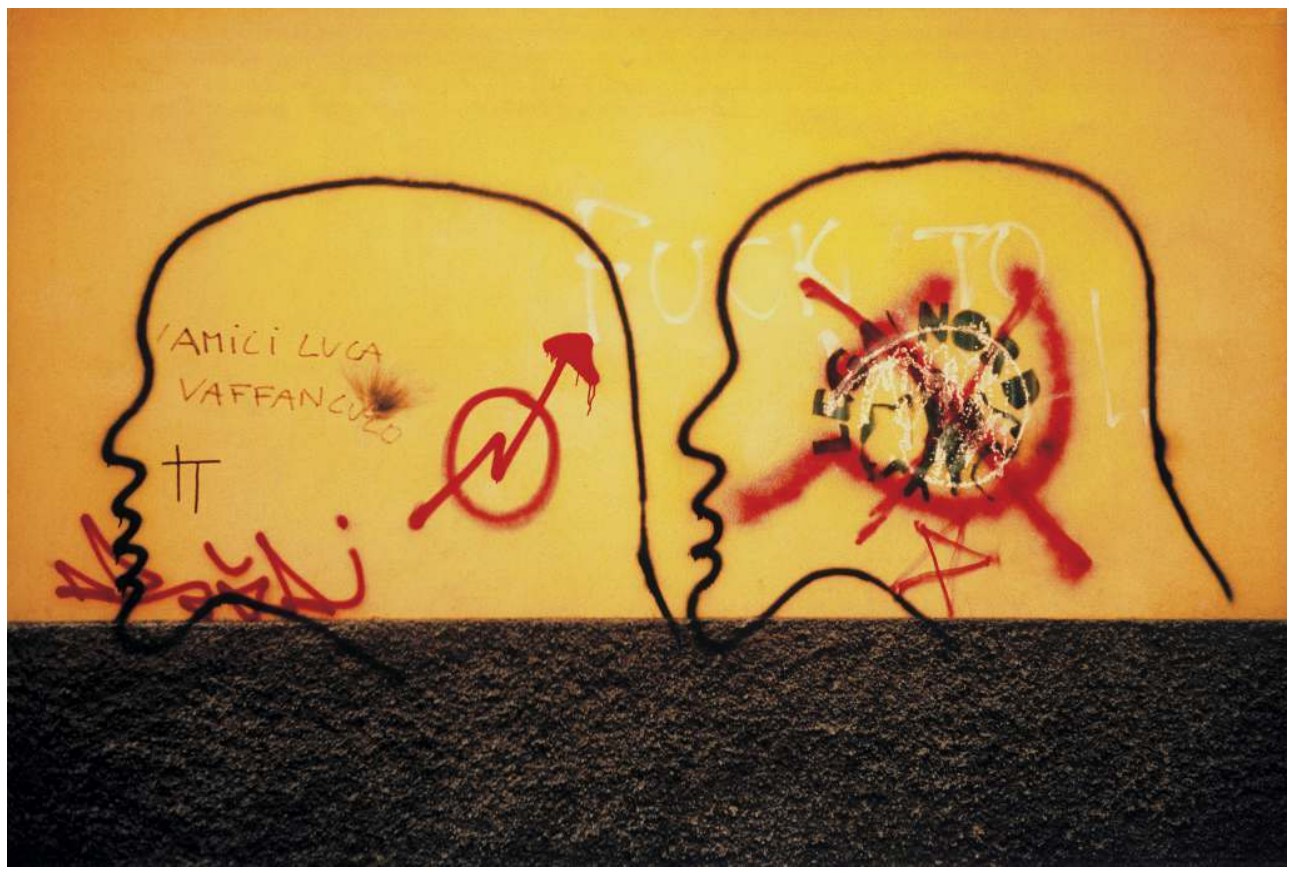

Plus tard, il comprendra que certains graffeurs prenaient sa tête nue pour le symbole de la tête rasée des skinheads néo-nazis. Cette expérience est comme une révélation pour Zhang Dali, pour qui le graffiti peut donc donner naissance à un échange mais cela reste utopique puisque, derrière le graffiti pratiqué en Italie, se cachent des différences culturelles et linguistiques qui empêchent de communiquer?

De retour en Chine en 1995, Zhang Dali choisit de se réinstaller à Pékin. Et il continue le même geste dans cette ville en pleine mutation entre construction démesurée et démolition de vieux quartiers à grande échelle. Trois ans après, le nombre des têtes qu'il a dessinées est de 2000 depuis le centre-ville jusqu'au troisième périphérique. Souvent, à côté de la tête dessinée, l'artiste inscrit les signes AK-47 ou 18K.

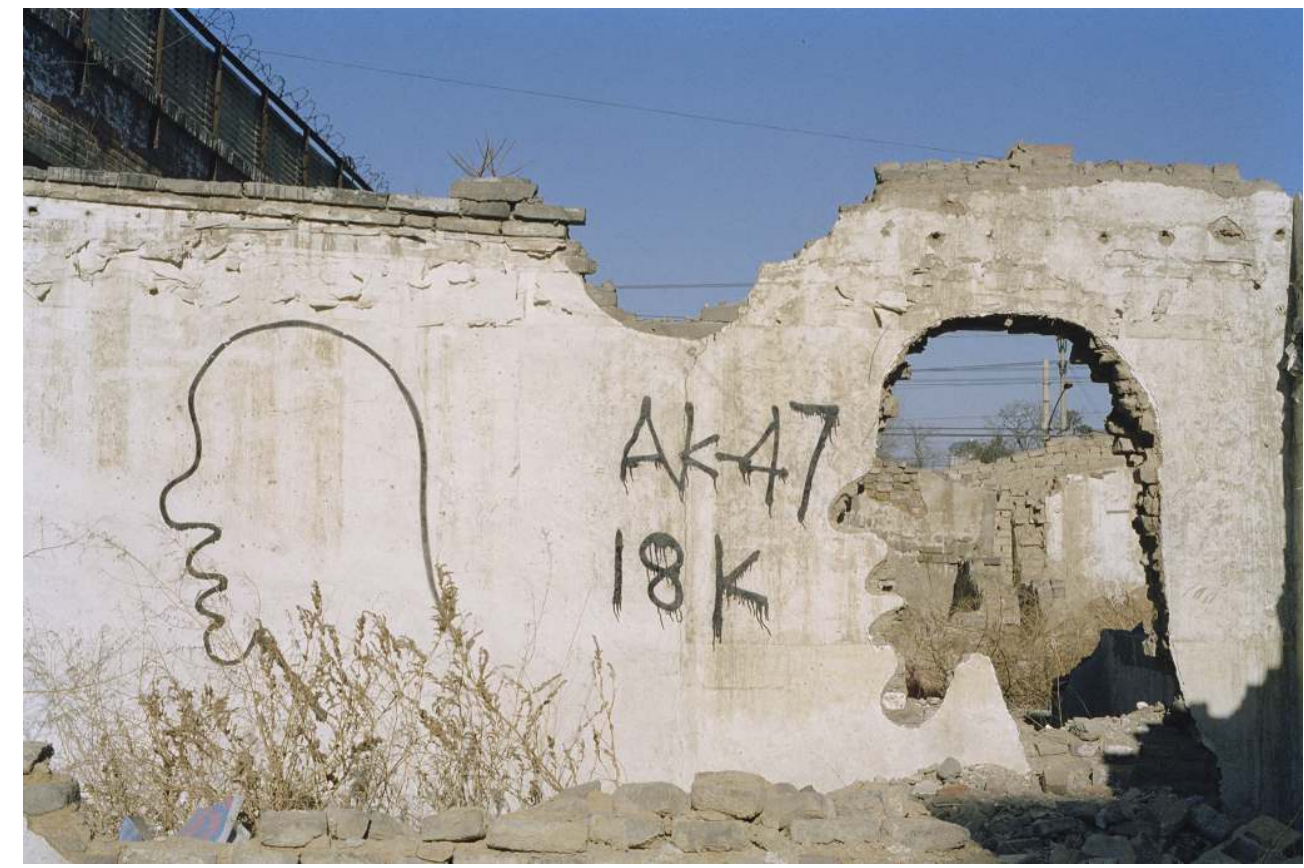




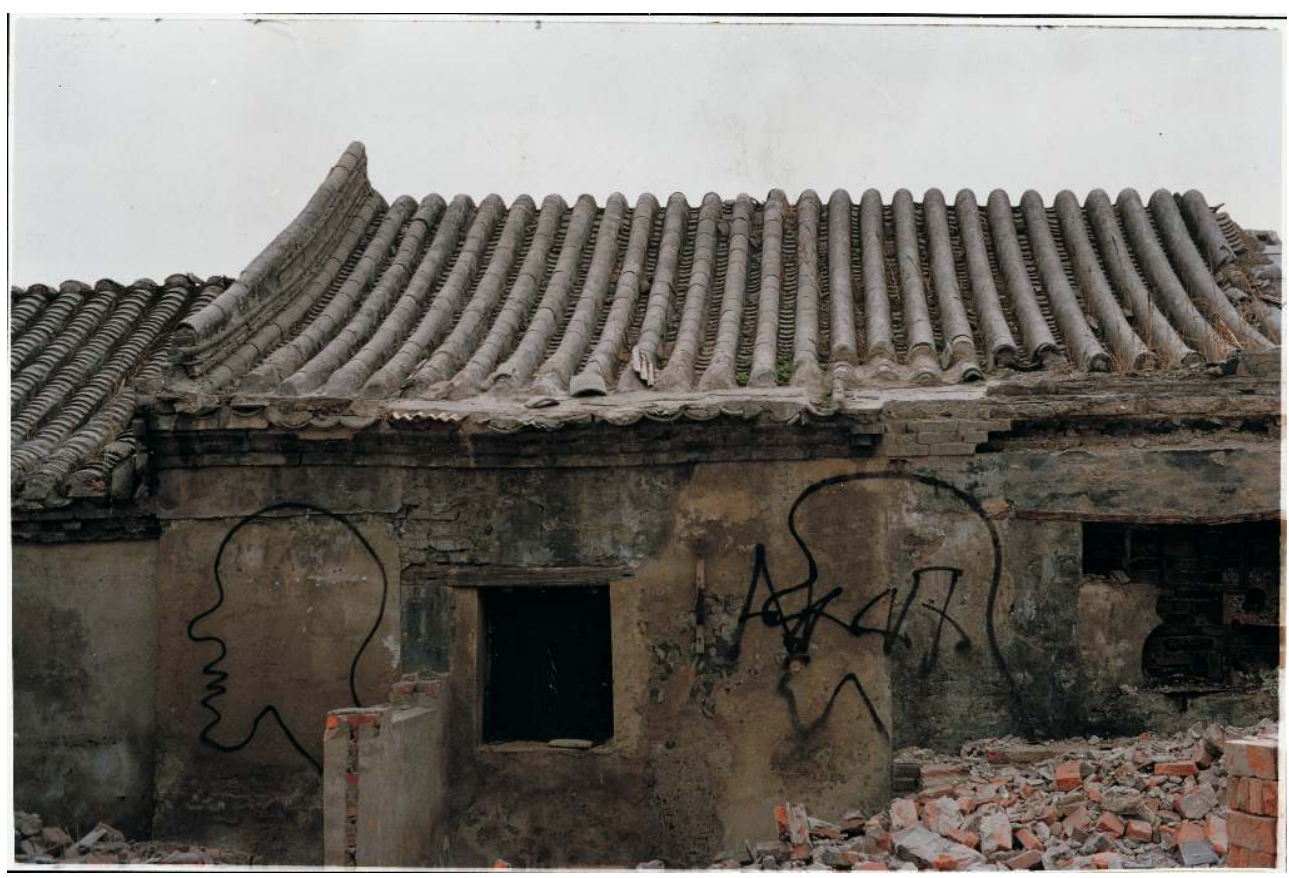

10 Les deux formules manifestent, selon l'artiste, la violence et la richesse qui résument la ville vouée au changement radical. Pour ne pas être pris, Zhang Dali se comporte comme un guérillero et se promène en vélo dans la nuit pour réaliser ses têtes nues dont le dessin ne dure que deux ou trois secondes. Dans la journée il se cache dans la foule pour écouter attentivement les commentaires des habitants et il les collecte.

11 En 1996, les graffitis de l'artiste suscitent des réactions et ces dernières sont recueillies et publiées dans le magazine intitulé Street (Jiedao $\left.{ }^{8}\right)$. L'article considère que l'ensemble des graffitis est vulgaire, comme une imitation d'un art occidental décadent. Les auteurs de cet article recueillent les opinions des habitants, dont un professeur universitaire, un restaurateur local, un passant, un travailleur migrant, un membre du comité résidentiel local, un policier, etc. La critique la plus dure provient de ces deux derniers qui demandent de capturer tout de suite l'auteur des graffitis tandis que les autres ne donnent pas non plus des opinions positives. Quant à Zhang Dali, il continue son geste puisque ces opinions ne sont pas un verdict officiel et il se trouve encore plus excité par ces retours négatifs".

12 À partir du début de l'année 1998, quelques journaux et magazines culturels pékinois lancent les débats sur les graffitis de l'artiste ${ }^{10}$. Ceux-ci réussissent à susciter des débats non seulement concernant les problèmes sociaux et politiques tels que la violence radicale subie par les habitants et la limite de leur patience, la politique environnementale et le projet urbain, mais aussi concernant quelques problématiques artistiques comme la question d'un art public et sa relation avec l'art de la performance, la liberté d'expression de l'artiste et sa responsabilité, etc. Tous ces débats sont en effet inséparables de l'ensemble du changement complexe de la ville qui est en train de devenir une métropole globale. C'est là que l'on voit enfin l'efficacité de l'artiste, avec la simplicité de son inscription sur la ville en pleine période de mutation : le graffiti révèle les problèmes divers touchant l'ensemble de la société chinoise qui chemine entre tradition et modernité, entre la voie socialiste et la voie capitaliste.

13 C'est après ces publications que Zhang Dali abandonne son anonymat et se joint à la discussion. Il donne sa première interview en mars 1998 en déclarant qu'il s'intéresse 
avant tout à la vie urbaine, son environnement changeant et la violence de la démolition ${ }^{11}$ . L'artiste explique que la tête nue est son autoportrait à travers lequel il veut engager un dialogue avec la ville. Cependant ce dialogue ne se limite pas à laisser une trace sur la matérialité architecturale de la ville ; il prétend aussi interpeller les habitants qui ont des regards très différents. Dans les conversations recueillies par l'artiste lui-même on trouve que la tête nue est considérée parfois comme le symbole de la mafia, parfois comme le marquage indiquant la limite de la démolition réalisé par des travailleurs-migrants ${ }^{12}$. Selon l'artiste, c'est seulement à travers toutes ces réinterprétations que le graffiti prend son sens et que le dialogue peut avoir lieu. Et c'est aussi à travers les réactions des habitants que l'artiste peut mieux connaitre leur mentalité ${ }^{13}$.

Entre 1998 et 1999, l'artiste continue son geste notamment sur les sites des quartiers et des maisons démolis. Trois aspects peuvent résumer les traits des œuvres de cette période selon le critique d'art Wu Hung ${ }^{14}$. Le premier est celui du rapport entre construction et destruction : les têtes nues sont inscrites sur le site de démolition entouré fort souvent par un environnement de gratte-ciels en construction.

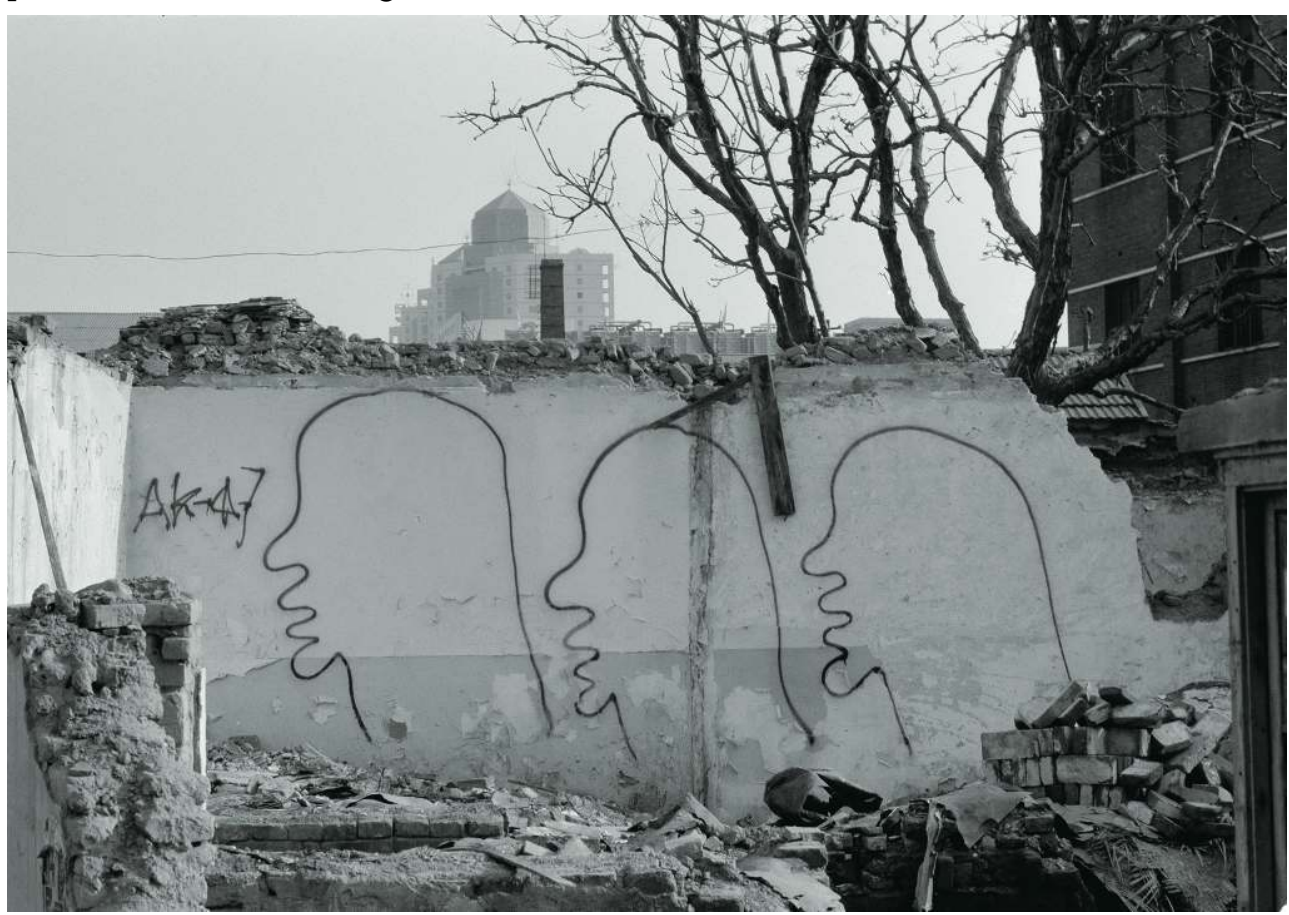

Le deuxième aspect est celui du rapport entre la démolition et la préservation. Toujours à travers les photos on constate qu'à l'intérieur du dessin de certaines têtes il a ménagé un trou à travers lequel on aperçoit le toit d'une ancienne architecture préservée et protégée par l'État. 


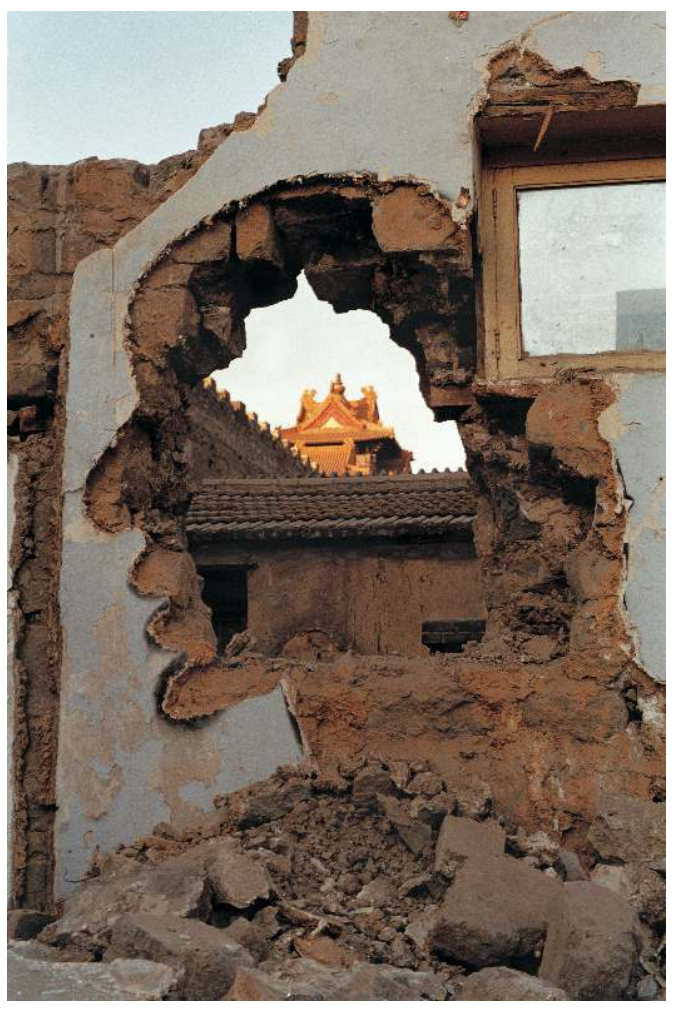

16 Quant au troisième, il interroge directement sur le statut de l'art du graffiti qui révèle le rapport entre un art central dominant et un art périphérique. Une photo montre une rangée de têtes nues dessinées sur un mur blanc démoli à moitié au-dessus duquel notre regard rencontre le fameux National Art Museum of China.

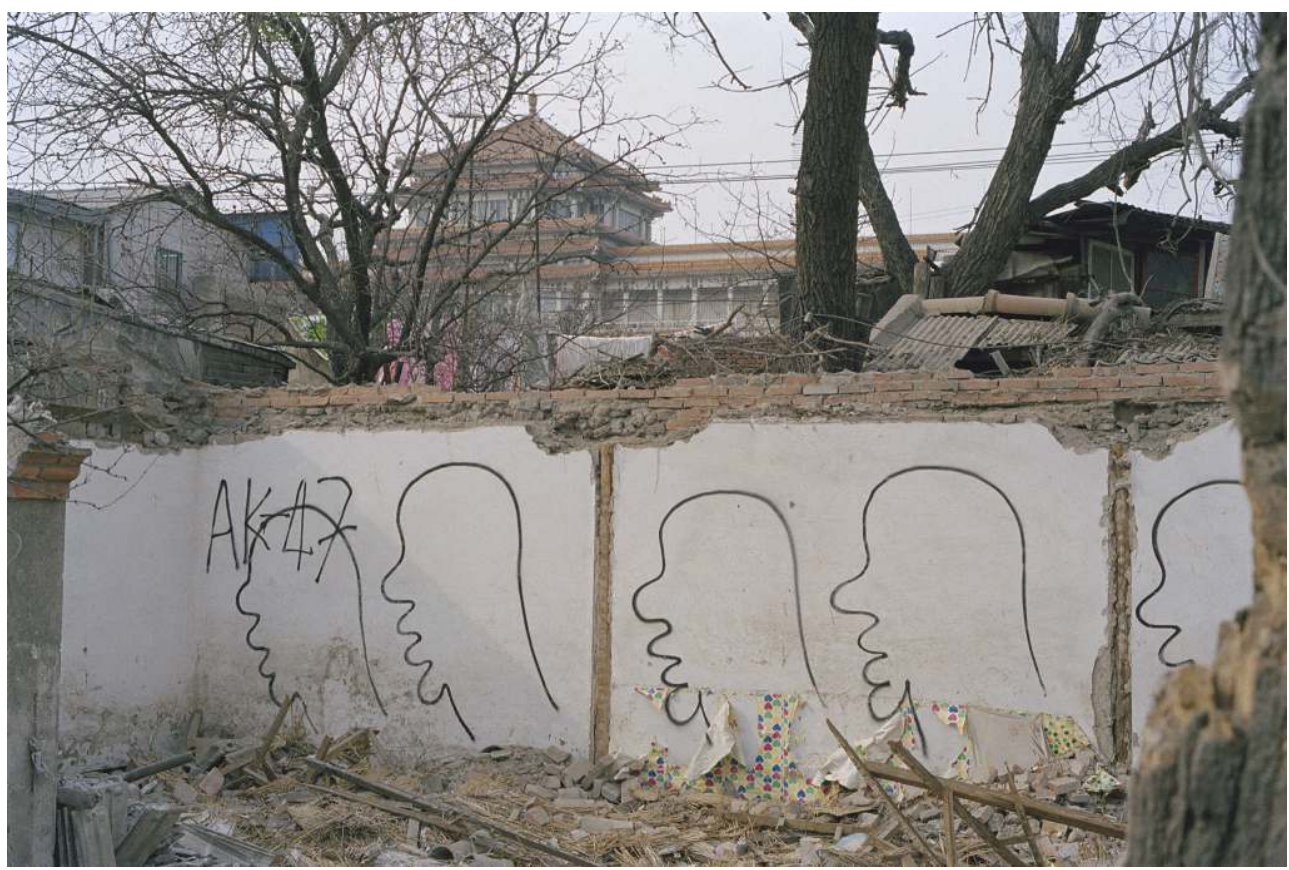

L'œuvre affirme, semble-il, la position de l'artiste dans un art périphérique. Il est conscient que «ses têtes» finiront par disparaitre avec la démolition du mur et la nouvelle construction. 


\section{L'état actuel du graffiti}

18 Zhang Dali est considéré comme le père de l'art du graffiti en Chine par certains historiens et critiques d'art comme Gao Minglu, Wu Hung et Leng Lin ${ }^{15}$. Cependant, aucun critique influent en Chine ne s'intéresse aux graffeurs d'aujourd'hui. Très probablement, ils pensent que les contenus des graffitis issus des jeunes générations ne relèvent pas suffisamment de problématiques méritant l'attention comme celles trouvées chez Zhang Dali. De plus, l'inspiration des jeunes graffeurs provient directement de la culture Hiphop, une grass-root culture (Caogen wenhua) pour la majorité des historiens d'art orthodoxes qui n'a ni les moyens ni l'envie de la déchiffrer.

19 Les graffeurs d'aujourd'hui forment un groupe composé de deux générations, celle qui est née après 1980 et celle d'après 1990. La majorité d'entre eux a reçu une formation d'art ou de design. En général, ils savent dessiner et considèrent que l'art du graffiti est un moyen d'expression à la mode par lequel ils peuvent manifester leur personnalité (gexing). L'un des premiers graffeurs de la génération d'après 1980 est un lycéen provenant de la ville de Guangzhou. Son pseudonyme est Dickid. Selon son récit personnel, en 1997, un jour, après avoir peint son skateboard à l'aide d'une bombe, il utilise le reste de la couleur pour écrire les mots en anglais « skate», "graffiti», « hip-hop » sur un mur en bas de chez lui. L'année suivante, il crée son site personnel CSN (qui cesse de fonctionner en 2006) pour diffuser les images de ses graffitis. En 2002, avec une autre graffeure nommée Sice et quelques lycéens, il crée le groupe MIG Crew (Made in Guangzhou), devenu l'un des premiers groupes du graffiti Chinois. Cette même année est apparu le FBL $\mathrm{Crew}^{16}$. Entre 2004 et 2005 surgissent des nouveaux graffeurs. Plusieurs sites géographiques aujourd'hui sont offerts à la visite pour découvrir cet art dans la ville de Guangzhou. On repère notamment deux terrains de basketball, celui de la Place de Kecunliying et celui de la Cité du sport Dongchuan; deux autres sites situés respectivement à la Guangzhou Academy of Fine Arts et au Lycée professionnel des Beaux-arts. On trouve également dans la même ville des graffitis dans des rues comme la rue Changgang, la rue de Beijing, etc.

Hong Kong ${ }^{17}$ joue aussi un rôle important dans l'influence de l'art du graffiti en Chine continentale. MC Yan (dont le vrai nom est Chen Guanren, né en 1971), par exemple, membre du groupe hip-hop cantonais LMF (Lazy Mutha Fucka), se considère comme le premier graffeur moderne de l'Asie. Selon certains médias, c'est lui qui introduit le graffiti dans des grandes villes près de Hong Kong comme Guangzhou et Shenzhen. Et c'est en effet dans ces dernières villes qu'est apparu d'abord le graffiti dans la Chine continentale.

21 Au début des années 2000, le graffiti réalisé par la génération née après 1980 émerge dans des grandes villes comme Pékin, Shanghai, Wuhan, Changsha, Chongqing, etc.

À Pékin, plusieurs groupes apparaissent après l'an 2000 dont Kwanyin Clan, BJPZ, Soos, ABS (Around Bohai Sea, et d'autres, également connus, comme Active, Brilliant, Significant), TMM (The ManageMent), KTS (Kill the Streets), etc.

Fondé en juin 2006, le Kwanyin Clan ${ }^{18}$ est considéré comme le premier groupe de graffeurs à Pékin. Son nom provient de celui du bodhisattva de la miséricorde. Dans l'histoire du bouddhisme, seule l'image de Kwanyin se transforme progressivement de l'apparence d'un homme en celle d'une femme. Le groupe récupère le nom du plus célèbre des bodhisattvas vivant une transformation pour évoquer leur ambition de 
transformer l'art du graffiti de l'Occident en un art spécifiquement chinois. Pour avoir un effet visuellement chinois, il introduit non seulement une iconographie chinoise mais aussi s'inspire de la peinture traditionnelle de paysage, de sa composition, de sa couleur en noir et blanc et de son fond avec sa couleur de papier ancien.

Un autre groupe travaillant dans le même esprit dans le but de créer un art graffiti chinois est le groupe BJPZ, fondé en février $2007^{19}$. Son nom est un acronyme phonétique du Beijing penzi. Le verbe pen se traduit littéralement par le geste principal de la réalisation du graffiti: "spray ». Quant au mot $z i$, c'est un suffixe. Le nom penzi est couramment utilisé pour désigner les graffeurs notamment à Pékin. Les membres sont tous graphistes et s'occupent de design commercial notamment celui des skateboard decks. Et ils possèdent un studio pour travailler ensemble sur le graffiti. Chaque membre a une marque spécifique pour se distinguer l'un de l'autre. Li Qiuqiu est connu pour la série des tags graphiques 0528 et la tête dessinée de type chinois. Mo invente un personnage ayant deux incisives. Quant à Soos, il aime dessiner la forme de la méduse. Malgré leur ambition, à comparer leurs réalisations à celles du groupe Kwanyin, ils ne nous offrent pas forcément des formes renvoyant à la tradition chinoise.

Il existe aussi des graffeurs indépendants comme Moses, Taps et Shat, etc. A Pékin, en tout, on compte au moins cent graffeurs de l'âge de 18 ans à l'âge de 40 ans. La majorité d'entre eux a un travail fixe en relation avec l'art ou le design. On remarque également que les graffeurs chinois, comme ceux des États-Unis, créent leurs pseudonymes et développent par la suite leurs tags personnalisés. Plusieurs emplacements dédiés au graffiti à Pékin sont visitables : à l'extérieur du Beijing University of Chinese Medicine ( Yinghua east street); le «cinquième passage à niveau " de la ligne ferroviaire Jingbao, entre les $4^{\mathrm{e}}$ et $5^{\mathrm{e}}$ boulevards périphériques ; la Renda South Road ; l'intérieur de la Renmin University of China, l'intérieur de l'Advertising School of CUC (Communication of University of China). Il y a aussi deux sites qui se situent dans des espaces consacrés à l'art contemporain : la fameuse zone d'art contemporain, l'espace 798 et le Pingod Apartments (Pingguo shequ) situé au Today Art Museum.

Malgré les sites cités précédemment, il est toujours difficile de trouver un lieu pour réaliser une masterpiece dans des sites déjà occupés par les graffitis. On illustrera ce point avec l'exemple de BJPZ qui voulait réaliser une œuvre consacrée à la journée mondiale de la lutte contre le SIDA. Ils choisissent d'abord un mur dans une ruelle de Pékin autour de laquelle il y avait des travaux. Ils pensaient que le fait de réaliser leur projet dans ce genre de lieu ne pourrait pas susciter de protestations. Mais malheureusement lorsqu'ils passent à l'acte dans la soirée, les habitants du quartier viennent les en empêcher. C'est une des raisons pour laquelle, certains groupes collaborent avec des entrepreneurs privés qui ont besoin de décorations sur les murs de leurs locaux. Du coup, les graffeurs peuvent réaliser leurs œuvres de manière créative tout en gagnant de l'argent. En revanche, la plupart du temps c'est l'entrepreneur qui donne le sujet du graffiti au groupe. Le graffiti en Chine est ainsi devenu de plus en plus dépendant d'enjeux commerciaux et c'est bien le cas à Shanghai.

Dans cette immense ville, plusieurs groupes sont fondés à partir de 2005, parmi lesquels le Reload $\mathrm{Crew}^{20}$, Oop et Beast Mode ${ }^{21}$ sont les plus représentatifs. Le Reload Crew présente des styles variés inspirés du design, de la peinture murale, des dessins animés. Il collabore avec des marques de produits commerciaux comme BMW, Volvo, Nike, Converse, KFC, etc. À Shanghai, le fait de fonder un groupe est aussi un moyen de se présenter comme une petite entreprise capable de recevoir et de réaliser des commandes 
publicitaires. Le Reload Crew non seulement se charge de réaliser des commandes mais aussi agit comme un agent commercial pour vendre les outils professionnels du graffiti provenant de l'étranger. Ces deux activités essentielles permettent au groupe de s'assurer des revenus afin de promouvoir leur art et de transmettre leur savoir-faire aux collégiens et lycéens. Quant aux membres des groupes Oop et Beast Mode, ils collaborent notamment avec des marques de voiture. En 2012 ils travaillent pour le "Chevrolet event » qui leur permet de peindre sur la voiture, alors que cela était impossible en Chine.

Le mur du graffiti le plus long et le plus complet à Shanghai, démoli en 2013, fut celui qui encerclait le fameux site d'art contemporain M50 Art Space. Peint sur le mur extérieur, l'ensemble des graffitis mesurait 600 mètres de long. En 2004 une entreprise privée achetait une partie de ce terrain près de la rivière de Suzhou mais les travaux prévus n'eurent pas lieu à cause de problèmes dus à la relocalisation des habitants qui vivaient là. Des jeunes graffeurs locaux émergeants de cette époque et les graffeurs étrangers commencèrent à dessiner sur ce mur qui était devenu inutile. A part le mur de M50 qui est aujourd'hui détruit pour laisser la place à de nouveaux projets commerciaux, il reste encore quelques sites de graffitis: celui d'une friche industrielle, située dans la rue Lingshi - elle est connue sous le nom de l'entreprise Shanghai Graffiti Park, fondée par le Reload Crew; celui de la rue Wenshui qui offre un mur d'une surface de 1000 mètres carrés est dédié uniquement aux images des dessins animés chinois; celui qui se situe dans un terrain de basket-ball nommé Park Rucker, portant le même nom que celui de New York. C'est un terrain privé dans lequel on présente les graffitis du Oops Crew.

À travers ce bref parcours consacré à trois grandes villes, on peut dire que la nouvelle génération des graffeurs chinois met avant tout l'accent sur un style original dans un souci d'expression personnelle. Ils sont comme ceux de l'Occident qui réalisent des tags, des Throw-up, du Wild style, des bombes, des 3Ds, etc. L'inspiration de leurs sujets principaux provient souvent du monde des dessins animés, parfois d'un monde imaginaire dont les personnages ont souvent un aspect "branché » provenant de la culture hip-hop. La démarche personnelle de création est parfois improvisée, parfois préparée dans le Black Book. S'il s'agit d'une masterpiece collective du groupe, cela dépendra de la démarche spécifique de chaque groupe. Pour les membres de BJPZ, ils tâchent d'abord de trouver un sujet, et par la suite chacun réalise de son côté le croquis à l'avance dans son Black Book, puis les membres se réunissent pour rassembler tous les croquis dans une seule composition, et enfin ils choisissent un lieu pour la réalisation sur mur. Tandis que pour les membres de l'ABS Crew, ils procèdent d'une manière improvisée.

30 Face à cette culture du graffiti qui chemine entre la liberté d'expression et les contraintes d'un monde commercial, le gouvernement chinois et les autorités locales adoptent une position assez ambigüe. Le fait de gribouiller sur un mur est illégal mais l'amende encourue n'est pas très élevée. En effet, tout dépend du contenu du graffiti ; s'il ne s'agit pas d'un sujet politiquement sensible, le fait de dessiner sur un mur est considéré comme un comportement tolérable. Souvent, dans chaque ville, c'est le bureau de gestion urbaine (Chengguanju), et non la police, qui traite le problème du graffiti pour maintenir la propreté de la ville. En attendant, les gouvernements locaux chinois semblent promouvoir cet art et les sites du graffiti cités plus haut ont reçu leur agrément.

31 Cette politique apparaît clairement dans la préparation des Jeux Olympiques. En 2008, avant cette manifestation, pour que la ville de Pékin ait une apparence nette et propre, la municipalité a effacé des graffitis visibles dans des lieux non autorisés. Cependant, un 
nouveau mur, nommé Mur culturel des Jeux Olympiques de Pékin, est construit d'une manière officielle près du 798 Art District pour les graffeurs afin de promouvoir les Jeux. Le but de ce projet consiste à donner une image positive et dynamique de la ville de Pékin ainsi que de la nation. C'est le même cas pour la ville de Chongqing qui possède la plus grande surface de graffiti de Chine, (et peut-être du monde...). Nommé Huangjueping Graffiti Art Street, cette rue mesure 1,25 kilomètres de long et présente une surface de 50000 mètres carrés offerte aux artistes. L'ensemble du projet a été réalisé entre 2006 et 2007. Son but consistait à embellir un quartier dégradé et sale et pourtant très visité par des étrangers grâce à la présence du Sichuan Fine Arts Institute rendu célèbre par les artistes Luo Zhongli, Zhang Xiaogang, Wang Guangyi, très cotés sur le marché de l'art mondial. Afin de donner une bonne image de ce quartier, Luo Zhongli, fort de sa notoriété, et en tant que directeur de cet institut, a eu l'idée de donner un nouveau visage au quartier. On le voit, c'est une démarche officielle qui prétend forger l'image de la ville de Chongqing, à la fois pour lui donner un look artistique dynamique et pour promouvoir l'économie de la ville. Dans le même esprit, on peut aussi citer la grande compétition nationale de graffiti organisée à l'occasion de l'Exposition Universelle de Shanghai en 2010, patronnée par les CCTV4 et CNTN. La compétition a pour but de susciter la création d'images de la ville du futur. Le graffiti, considéré auparavant comme le résultat d'une démarche assimilée à un vandalisme venant de l'Occident, se transforme en un outil idéal pour faire de la propagande au service du gouvernement chinois. De plus, les jeunes graffeurs deviennent un sujet important pour les médias officiels. En 2012 la Télévision Centrale de Chine (CCTV) diffuse une série de documentaires intitulée Nouvelle jeunesse qui est censée éduquer la jeunesse. On y voit en particulier deux épisodes qui sont respectivement consacrés aux groupes BJPZ et ABS. Chaque documentaire qui dure à peu près une vingtaine de minutes montre à quel point les jeunes graffeurs font des efforts inlassables pour se perfectionner dans ce nouveau métier et comment ils consacrent leur temps, leur énergie et leurs revenus, parfois modestes, à aider les personnes en difficulté. Les deux documentaires sont réalisés d'une manière très réaliste avec des interviews très pertinentes et touchantes.

\section{Les cas exceptionnels}

\section{Le graffiti de la ruelle Kangding à Shanghai ${ }^{22}$}

Devant cette apparence positive de l'art du graffiti géré par l'État se cache aussi une face sombre. À Shanghai, fin janvier 2015, dans un chantier de démolition d'un vieux quartier nommé ruelle Kangding, apparaît une dizaine de peintures murales réalisées par l'artiste chinois Shi Zheng et l'artiste français Seth (Julien Malland). L'artiste Shi Zheng, peintre et photographe, exerce son art depuis 20 ans et a déjà fait des expositions personnelles. Quant à Seth, c'est l'un des graffeurs français le plus connu à l'étranger qui voyage et réalise ses graffitis dans le monde entier. Sa page personnelle sur Facebook rassemble presque 300,000 fans. Ses personnages sont souvent directement inspirés des habitants locaux. Et ses compositions sont en général adaptées avec intelligence aux lieux où il opère ${ }^{23}$.

Pour cette réalisation de la ruelle Kangding, Seth est parti de croquis de sept ou huit enfants dans son carnet. Les traits de ses personnages possèdent des caractères chinois : les nattes, les foulards rouges, les yeux bridés, etc. 

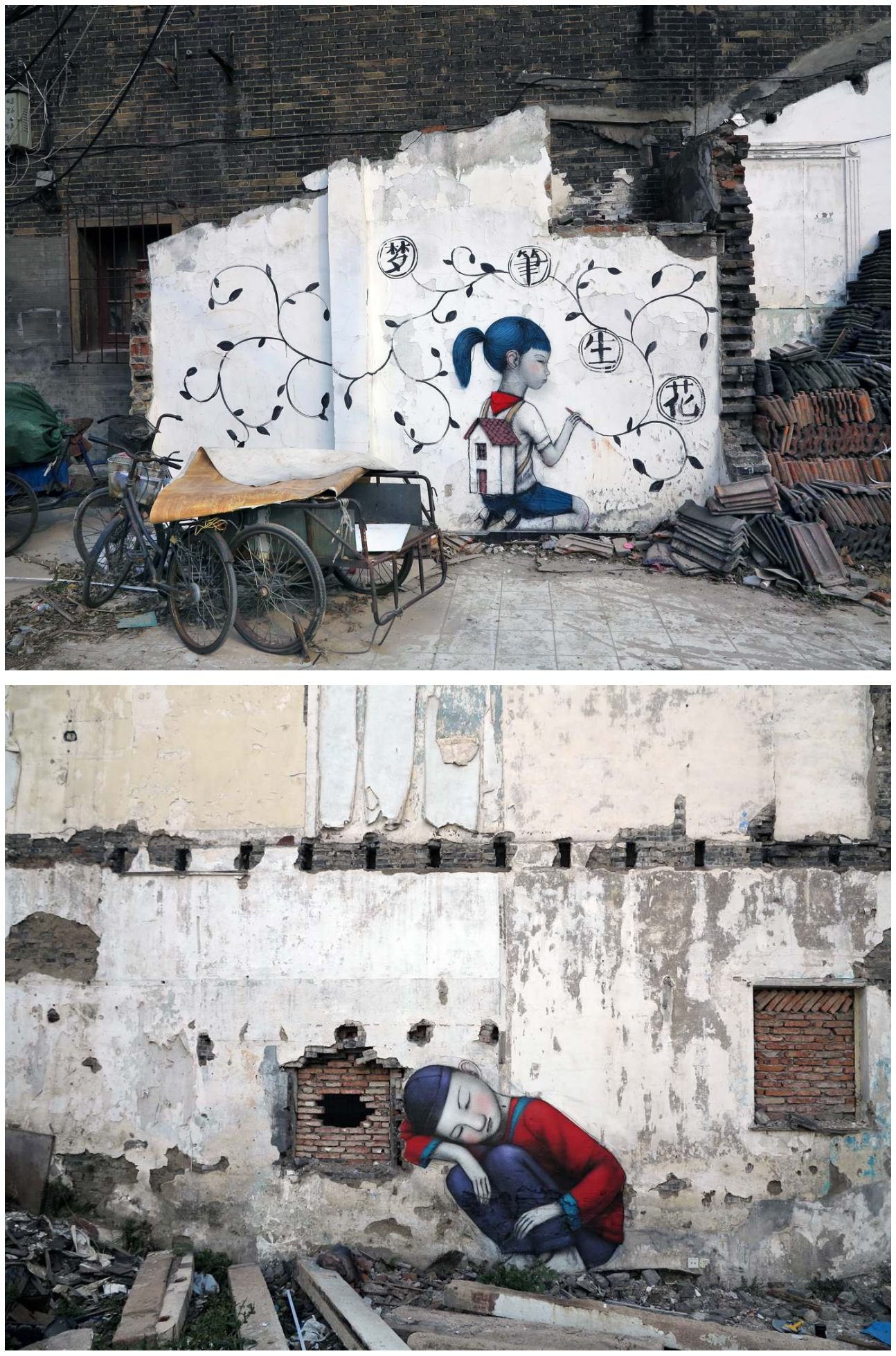


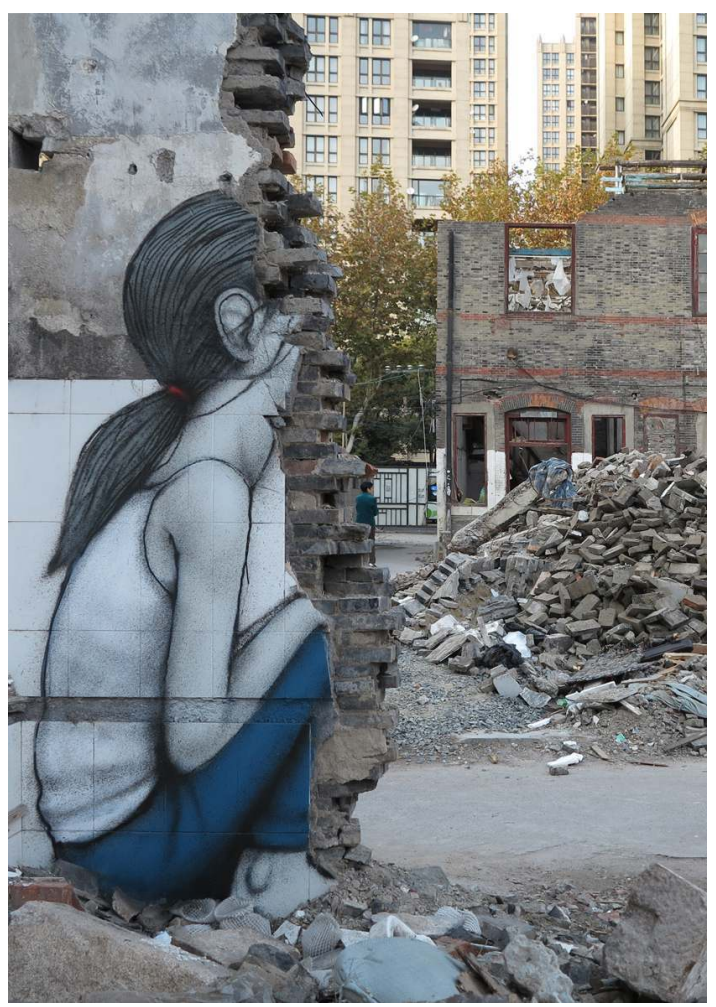

À propos de la relation entre les personnages et le lieu, Seth ajoute ceci :

Ce qui me plaisait dans le fait de peindre à ces endroits est de témoigner et de parler de la Chine en transformation. La disparition de quartiers traditionnels qui font place à des quartiers nouveaux proches de la modernité occidentale. En jouant avec l'architecture et l'aspect des supports j'essaye de créer un univers poétique et nostalgique, basé sur le contraste entre la destruction et des personnages doux, qui parlent à chacun de manière différente ${ }^{24}$.

Seth et son collaborateur chinois ont bien saisi le sujet de cette série : la démolition et la relocalisation. J'en donne ici quelques descriptions : une peinture représente une fillette portant dans ses bras une maison, avec des larmes sur son visage ; une fille et un garçon, tous deux portant des foulards rouges, se tiennent face à face de part et d'autre de l'emplacement d'une porte disparue qui coupe leurs visages; une fille svelte met son visage dans le trou d'un arbre comme si elle ne voulait pas voir la scène de la démolition de son quartier natal. Autour d'elle Shi Zheng ajoute des jeunes rameaux entrelacés sur lesquels poussent quelques bougeons rouges portant le sinogramme "démolir»(拆) encerclé. Ce symbole est souvent utilisé dans le processus de la démolition pour prévenir les habitants. Les vers poétiques de Bai Juyi (772-846) sont aussi insérés par l'artiste chinois : « Le feu de la prairie ne saura détruire l'herbe. Elle repousse de plus belle sous le vent du printemps. » À propos de cet ajout littéraire, Shi Zheng donne cette explication : "la démolition fait naître inévitablement des sentiments de regret, mais elle est également porteuse d'espoir, d'où le sens des herbes qui repoussent ${ }^{25}$.» 


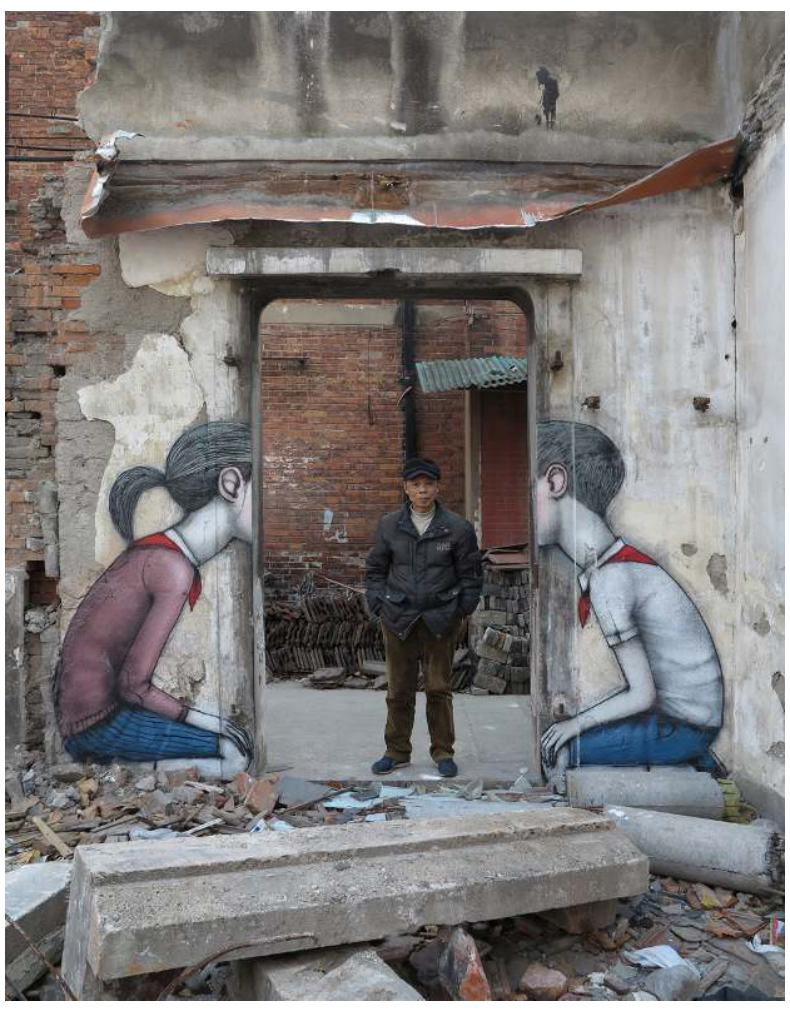

36 Deux autres vers d'un poème de Meng Haoran (691-740) intitulé Aube du printemps que l'on apprend par cœur à l'école primaire, sont calligraphiés au-dessus d'une fille endormie : «Toute la nuit, bruit de vent et de pluie, Qui sait combien de fleurs ont dû tomber ${ }^{26 !} »$.

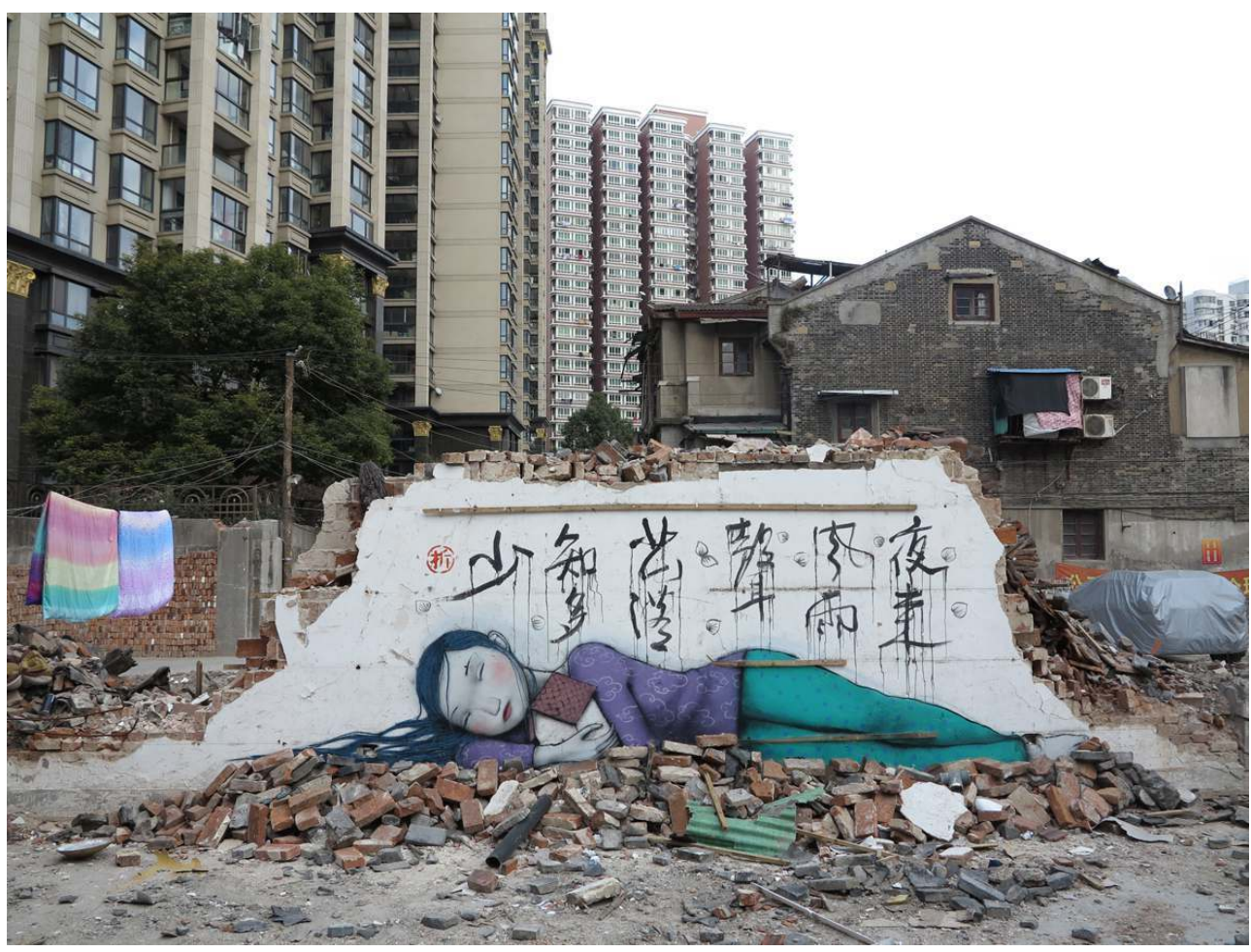

37 Une belle métaphore semble dire combien de maisons vont tomber dans cette opération de démolition. Une écolière montre au public son dessin d'une maison sur laquelle est barré le signe « démolir ». À côté est écrit par Shi Zheng: "La poésie est partout, toute 
chose a sa splendeur. » «Même les gravats, explique l'artiste, ont une beauté qui mérite d'être mise en valeur, c'est ainsi "toute chose a sa splendeur ${ }^{27 "}$ ". Dans la plupart des cas, c'est Seth qui réalise d'abord une image et Shi Zheng insère des sinogrammes et des vers calligraphiés. Les deux artistes, malgré la barrière linguistique, ont pu travailler ensemble car le courant est passé entre les deux ${ }^{28}$. L'ensemble des graffitis a renforcé l'atmosphère nostalgique et mélancolique de ce lieu vouée à la démolition.

C'est à travers les réseaux sociaux que les graffitis de la ruelle Kangding se sont faits connaître des habitants de Shanghai. Ils attirent alors de nombreux d'admirateurs - de deux à trois cents spectateurs par jour. Mais, à partir du 24 janvier, devant chaque peinture est hissée une pancarte avec ces mots: «site de construction, interdiction d'entrée». Par la suite les peintures sont rapidement détruites "pour raisons de sécurité" même avant la démolition complète du quartier. Et cela suscite le mécontentement de certains médias :

Les graffitis réalisés dans un quartier promis à la démolition finiraient de toute façon par disparaitre. Mais alors même qu'ils sont en train d'être appréciés par le spectateur, la pioche les réduit en poussière. Cette violence du pouvoir et cette volonté de réduire au silence sont évidemment... bien discutables ${ }^{29}$.

On voit ici que la tension est née du gouvernement local qui agit pourtant d'une manière prudente et efficace face à cette réputation inédite du graffiti sachant qu'il risque de créer des polémiques au sujet des destructions. Or c'est un sujet très sensible rappelant immédiatement le souvenir douloureux des expulsions forcées, de la corruption du gouvernement local et de la réponse désespérée de certains habitants qui finissent parfois par s'immoler par le feu. En conséquence, le gouvernement local n'a pas attaqué directement l'artiste chinois ni jugé frontalement le contenu des graffitis mais donné seulement le prétexte de la sécurité pour les effacer. Ce qui est conforme à ce genre de politique répressive qui s'applique le plus souvent à taire le véritable objet de la censure.

\section{L'incident de la " graffiti girl »}

Une autre affaire que je voudrais évoquer a eu lieu à Hongkong. En avril 2011 lorsque le célèbre artiste chinois Ai Weiwei (né en 1957) est arrêté par la police à Pékin juste avant son départ pour Hong Kong sous le prétexte d'une infraction d'évasion fiscale, de nombreux portraits de l'artiste sous forme de graffitis au pochoir apparaissent dans les rues de Hong Kong. Les portraits sont accompagnés d'un slogan tantôt en anglais tantôt en chinois : «Who is afraid of Ai Weiwei ?»; « Shei pa Ai Weiwei ?». En effet, Ai Weiwei est mondialement reconnu pour ses actions et ses œuvres qui critiquent le gouvernement chinois et lui créent de nombreux ennuis. Par exemple, pour sa rétrospective intitulée "So Sorry » à Munich (oct. 2009 - jan. 2010), l'artiste réalise son installation Remembering qui consiste à exposer 9,000 cartables d'enfants sur la façade de la Haus der Kunst. Il le fait pour rendre hommage aux enfants qui sont mort en 2008 pendant le tremblement de terre de la province du Sichuan, sous les décombres des écoles. Les bâtiments ne respectaient pas les normes antisismiques à cause de la corruption locale.

L'image du pochoir d'Ai Weiwei se trouve partout dans Hong Kong, notamment dans le district du Sheung Wan, célèbre pour ses galeries d'art, ses antiquaires et le centre financier de la ville. L'auteur de cette image est une graffeuse de 22 ans appelée Tang Chin, connue aussi sous le nom Tangerine, dévoilé plus tard par les médias. Le but de son 
geste consiste à dire que la détention de l'artiste continental chinois touche aussi les hongkongais. Dans une interview, Tangerine déclare :

Il [Ai Weiwie] est l'un des plus importants artistes contemporains du monde. Et s'il peut être arrêté, cela veut dire qu'il n'y a pas d'identité derrière laquelle on peut se cacher : le fait d'être un citadin hongkongais ne peut plus nous aider... Il n'y a plus de bouclier à l'encontre de la puissance brute de ce pouvoir qui essaie de nous engloutir ${ }^{30}$.

Malgré la politique mesurée due à la règle "Un pays deux systèmes » (yiguoliangzhi), proposée et appliquée à Hong Kong par le gouvernement chinois après son retour en 1997, la force de la centralisation du pouvoir ne cesse d'agir d'une manière calculée et envahissante. Le geste de Tangerine n'est pas seulement une manière de soutenir l'artiste, mais aussi d'éveiller la conscience des hongkongais afin de susciter la discussion publique sur la situation actuelle des droits de l'homme à Hong Kong. Par le bouche-àoreille, par les médias, l'image du pochoir d'Ai Weiwei suscite des débats concernant notamment la démocratie à Hong Kong. Face à la montée de la conscience politique, la police locale décide de classer cet incident dans unusual case - cas inhabituel. D'habitude, dans cette ville un graffeur est condamné pour vandalisme à trois mois de détention. Mais cette fois-ci, le cas de la graffiti girl, nom inventé par l'un des médias, est étudié dans l'administration judiciaire par une équipe spécialisée dans les crimes graves qui traite des enlèvements et des assassinats. Aujourd'hui elle est recherchée par la police sous le coup d'un mandat d'arrêt et risque jusqu'à dix ans de prison. Le 15 avril 2011 à minuit, la graffiti girl lance un appel à continuer la diffusion de l'image du pochoir d'Ai Weiwei. Le combat continue. Le 23 avril plus de 2500 personnes manifestent dans la rue pour la libération d'Ai Weiwei. Le 29 avril, l'image du pochoir d'Ai Weiwei est projetée par un homme de 25 ans appelé A Ming sur les murs de neuf endroits différents de Hong Kong, y compris sur le mur du Police Headquarters du quartier de Wan Chai et sur celui du bâtiment des Chinese People's Liberation Army Forces cantonnées à Hong Kong.

De même, l'image du pochoir d'Ai Weiwei est aussi apparue en Chine continentale. Le 18 avril 2001 environs 35 images de l'artiste sont exposées dans le tunnel piétonnier souterrain de la ville de Hefei dans la province d'Anhui. Chaque image est accompagnée d'une phrase : «C'est une meilleure époque ». Un journal local publie un article intitulé «C'est de l'art ou ce sont des ordures ${ }^{31}$ ? » dans lequel il n'y a ni le nom de l'artiste ni l'explication de la phrase qui est, en effet, tirée d'une chanson appelée « Le peuple n'a pas besoin de liberté » composée par le chanteur folklorique indépendant Li Zhi. Le journal déclare que les passants sont plutôt choqués non seulement par les images inconnues, mais aussi par leur réalisation inhabituelle. L'article ne publie aucune image de cet incident. Cependant, le journaliste laisse une description littéraire et suffisamment précise du pochoir d'Ai Weiwei pour que l'on puisse deviner facilement qu'il s'agit de cet artiste. On peut penser que le journaliste a fait exprès d'éviter de prononcer le nom de l'artiste pour ne pas toucher directement un sujet politique sensible. Vers $11 \mathrm{~h} \mathrm{du}$ matin, les images et les phrases sont effacées sous l'ordre du bureau local de gestion urbaine. Cependant, l'image du pochoir d'Ai Weiwei continue à apparaître en Chine. Deux jours plus tard on la trouve dans une station du métro de Pékin. Le 25 avril, on la trouve dans un "village d'art" près de la cité universitaire de Guangzhou dans la province du Guangdong. Mais l'ampleur de cette continuation continentale n'a pas suscité autant de discussions que les évènements de Hong Kong. comme un dissident anti-gouvernemental, tandis qu'en Chine continental, son rôle restait 
ambigu pour le grand public. Au début, Ai Weiwei est apprécié par l'État lorsqu'il revient en Chine en 1993. Artiste, architecte et fils du grand poète chinois Ai Qing (1910-1996), il est invité par le gouvernement pour réaliser le design de l'architecture du Stade national de Pékin nommé le Nid d'Oiseau. Mais, comme on le sait, plus tard il se retire du projet en marquant une rupture avec le gouvernement. Quant à l'appréciation portée sur Ai Weiwei dans le milieu intellectuel, cela reste aussi très partagé. D'un côté, pendant sa détention, il est soutenu par le journal The Southern Daily qui conteste son arrestation. De l'autre côté, il est critiqué dans le milieu artistique, comme le note Shelly Kracier :

C'est surprenant pour moi de savoir que beaucoup de mes amis chinois libéraux (c'est-à-dire les critiques du système du gouvernement chinois, et qui sont antirépression et pour la liberté d'expression) et collègues pensent très peu à Ai. Ils le considèrent comme un showman pratiquant la démagogie, qui joue notoirement devant le portail d'entrée de la place Tiananmen en pointant son médius pour avoir l'adulation occidentale ${ }^{32}$.

Pour beaucoup de Chinois, c'est là un geste facile d'insulter le gouvernement tandis qu'en Occident, dans beaucoup de médias on le considère comme un geste héroïque qui s'oppose à la démolition par la force d'un village artistique à Pékin. Dans un tel contexte on voit qu'il est impossible de mener une grande lutte pour la démocratie et la liberté d'expression avec quelques graffitis représentant Ai Weiwei comme celle qui eut lieu à Hong Kong. C'est aussi pour cette raison que le gouvernement chinois n'a pas non plus fait beaucoup d'efforts pour rechercher les auteurs des graffitis. En revanche la graffiti girl hongkongaise est toujours considérée comme une criminelle recherchée par la justice.

\section{Conclusion}

Le graffiti en Chine est un outil idéal pour la propagande gouvernementale. Il s'intègre aussi parfaitement à des démarches d'art public commercial. Mais il est aussi un redoutable moyen d'expression capable de répondre rapidement à des enjeux politiques et sociaux quand le milieu s'y prête. C'est le cas à Hong Kong. À la différence de cette situation hongkongaise, dans un contexte chinois continental plus répressif, l'art du graffiti vient donner aux jeunes artistes les moyens d'exprimer rapidement et anonymement leurs sentiments; mais cela reste encore très timide. On peut constater que, dans le fil du geste de la graffiti girl, à Hong Kong, le combat continue pour la liberté d'expression et la démocratie, tandis qu'en Chine, l'image d'Ai Weiwei n'est plus un tabou, car on expose officiellement ses œuvres pour la première fois à la Galleria Continua ( Changqing hualang) et au Centre Tang d'art contemporain (Dangdai tangren yishu zhongxin) à Pékin (6 juin 2015 - 31 décembre 2015).

Quant au statut du graffiti, serait-il considéré comme un art un jour classé dans l'histoire de l'art contemporain chinois? Pour l'instant il est difficile de donner une réponse. A cause de son statut d'expression jeune et populaire, le graffiti développé après 2000 n'attire pas encore suffisamment l'attention des historiens et critiques d'art chinois. Face à cette culture populaire dans ses différentes manifestations - graffiti, mangas, Hip-hop etc. - devenue mondiale, il serait sans doute intéressant de placer la problématique de l'«art » du graffiti dans la perspective des Visual Studies qui échappent, dans leur vitalité actuelle, aux catégories peut-être trop limitées d'une histoire de l'art traditionnel. Evidemment, cela ne rendrait pas les choses plus simples, mais cela permettrait sans doute de passer plus aisément d'une pratique à l'autre en reconnaissant la part de 
créativité de chacune d'entre elles sans devoir sans cesse en référer à un modèle artistique aux contours hélas toujours plus flous...

\section{BIBLIOGRAPHY}

\section{Livres:}

Demiéville, P (1962) Anthologie de la poésie chinoise classique, Paris, Gallimard.

Gao, M.L. (2005) The Wall : Reshaping Contemporary Chinese Art, Beijing, Millennium Art Museum/ Buffalo, University at Buffalo.

Goodrow, G. (2014) Crossing China. Land of Rising Art Scene, Cologne, Daab, 2014.

Leng, L. (2000) Shiwo [C'est moi], Pékin, Zhongguo wenlian chubanshe.

Pan, L. (2015) Aesthetizing Public Space Street, Street Visual Politics in East Asian Cities, Intellect, The University of Chicago Press, Chicago.

Stewart, J. (1989) Subway Graffiti. An Aesthetic Study of Graffiti on the Subway System of New York City, 1970-1978, these de doctorat. New York University.

Wou, C.E. (1592) Le singe pèlerin, ou le pèlerinage d'Occident, traduit du chinois par Arthur Waley, 2003, Paris, Payot.

Wu, H. (2009) Contemporary Chinese Artists, Hong Kong, Timezon 8.

\section{Articles :}

Bo, M.X. (1998) « Inscriptions sur le mur ». In : Musical Life (Yinyue shenghuo bao), le 16 avril.

Commentateur du site Guangming Online (Guangming wang) (2015), « Bien traiter le graffiti consacré à la démolition, écouter avec respect la dernière chanson de la vieille maison ». In : Guangming Online. http://guancha.gmw.cn/2015-01/25/content_14631991.htm [5.11.15]

Dao, Z. (1998) « La logique de la culture d'un art de plein air ». In : China Readers (Zhonghua tushu bao), le 6 mai.

Fan, J. (2015) « Le graffeur Shi Zheng, la ruine contient de l'espoir ». In : Journal du soir de Xinmin ( Xinmin wanbao), Shanghai, le 25 janvier.

Hang, C. (1998) « Interview exclusive avec l'artiste du graffiti ». In : Life Time (Shenghuo shibao), le 18 mars.

Hang, C. (1998) « Portrait dans la rue, est-ce de l'art? ». In : Life Times, le 21 mars.

Jiang, T. (1998) « Se fixer sur le portrait de la tête nue de la ville de Pékin ». In : Bluesky Weekend (Lantian Zhoumo), le 17 mars.

Journaliste anonyme (2011), « C'est de l'art ou ce sont des ordures? » In :Journal du matin Jianghuai (Jianghuaichenbao), le 19 avril.

Kraicer, S. (2012) « Ai Weiwei : Never Sorry: Film review ». In : Chinese Cinemas. https:// chinesecinemas.wordpress.com/2012/12/19/ai-weiwei-never-sorry-film-review/ [5.11.15] 
Lim, L. (2011), « Hong Kong Graffiti Challenges Chinese Artist's Arrest” ». In : National Pubic Radio, Washington, D.C. http://www.npr.org/2011/05/04/135985475/hong-kong-graffiti-challengeschinese-artists-arrest [5.11.15]

Valiakka, M. (2011) « Graffiti in China - Chinese Graffiti? ». In : Copenhagen Journal of Asian Studies, Vol 29, 1, 61-91.

Yu, Z. (1998) « Le graffiti d'un inconnu dans l'avenue Pingan ». In : Beijing Youth Daily (Beijing qingnianbao), le 24 décembre.

Zhang Ming (2008), « l'Histoire du graffiti - "la tête nue" est devenue le symbole d'inscription du changement d'une ville ». In : Oriental Outlook (Liaowang dongfang zhoukan), 47, 82.

Zhao, G.M. (1998), « Pourquoi dessiner un portrait sur le mur? ». In : Beijing Youth Daily, le 18 mars.

\section{NOTES}

1. Minna Valiakka (2011), «Graffiti in China - Chinese Graffiti ?». In : Copenhagen Journal of Asian Studies, Vol 29, No 1, p. 61-91.

2. Norbert Kirbach (2014), «Street art in Hong Kong - Communication in Public Spaces ». In: Crossing China. Land of Rising Art Scene, edited by Gérard Goodrow, Cologne, Daab, chap. VII.

3. Pan Lü (2015), « Part 1: Carnival on the Street - Visual Order and its (Pseudo-) Reversion » et « Part II: Aura on (and beyond) the Street-Body, Community, and Media ». In: Aesthetizing Public Space Street, Street Visual Politics in East Asian Cities, Intellect, The University of Chicago Press, Chicago, p. 43-97.

4. La formule populaire « quelqu'un est passé ici » est inspirée de la mythologie du roi des singes, qui jadis laissa derrière lui l'inscription « Le Grand-Sage Egal-du-Ciel est passé ici » sur le médius de la main du Bouddha tout en croyant qu'il s'échappait de la paume de sa main. Voir Wou Cheng'en (1592), Le singe pèlerin, ou le pèlerinage d'Occident , traduit du chinois par Arthur Waley, 2003, Paris, Payot.

5. Jack Stewart (1989), Subway Graffiti. An Aesthetic Study of Graffiti on the Subway System of New York City, 1970-1978, thèse de doctorat. New York University, p. 146.

6. Devenue aujourd'hui Acadamy of Art and Design de Qinghua University.

7. Propos recueillis par Wu Hung (2000), «Zhang Dali's Dialogue, Conversation with the City ». In : Contemporary Chinese Artists, Hong Kong, Timezon 8, 2009, p.47-57., cit. p. 49.

8. Ibid., p. 47.

9. Propos recueillis par Zhang Ming (2008), «l'Histoire du graffiti - "la tête nue" est devenue le symbole d'inscription du changement d'une ville». In: Oriental Outlook (Liaowang dongfang zhoukan), n 47, p. 82.

10. Les articles sont: Yu Zhong (1998), «Le graffiti d'un inconnu dans l'avenue Ping'an ». In: Beijing Youth Daily (Beijing qingnianbao), 24 décembre ; Hang Cheng (1998), «Interview exclusive avec l'artiste du graffiti». In: Life Time (Shenghuo shibao), 18 mars ; Zhao Guoming (1998), «Pourquoi dessiner un portrait sur le mur ?». In: Beijing Youth Daily, 18 mars; Hang Cheng (1998), « Portrait dans la rue, est-ce de l'art?». In : Life Times, 21 mars ; Jiang Tao (1998), «Se fixer sur le portrait de la tête nue de la ville de Pékin ». In: Bluesky Weekend (Lantian Zhoumo), 17 mars; Bo Maxiu (1998), «Inscriptions sur un mur ». In: Musical Life (Yinyue shenghuo bao), 16 avril ; Dao Zi (1998), « La logique de la culture d'un art de plein air ». In : China Readers (Zhonghua tushu bao) 6 mai.

11. Leng Lin et Zhang Dali (1998), «Dialoguer avec la ville». In: C'est moi (Shiwo), Pékin, Zhongguo wenlian chubanshe, 2000, p. 166-179. 
12. Ibid.

13. Ibid.

14. Wu Hung, op. cit., p. 53-56.

15. Voir Gao Minglu, The Wall: Reshaping Contemporary Chinese Art, Beijing, Millennium Art Museum/Buffalo, University at Buffalo, 2005 ; Wu Hung (2000), op. cit., p. 47-57 et Leng Lin (1998), op. cit., p. 166-179.

16. L'acronyme FBL présente les trois mots chinois fei bao li signifiant «non-violence ».

17. Dans l'histoire de l'art du graffiti à Hong Kong il est nécessaire de citer Tsang Tsou Choi (1921-2007), dit le «Roi de Kowloon» qui, dès années 1950, employait la technique de la calligraphie chinoise pour inscrire notamment l'histoire de ses ancêtres dans les rues du quartier Kowloon [Neuf dragons]. Considéré comme un fou de son vivant, le «Roi de Kowloon» proclamait que le titre de ces ancêtres était celui d'Empereur de Kowloon et qu'il possédait ainsi une souveraineté légitime sur le territoire du quartier. C'était la raison pour laquelle il menait un combat à l'aide d'inscriptions calligraphiques pour lutter contre la colonisation de l'Angleterre. Aujourd'hui en Chine on nomme l'ensemble de ses inscriptions "œuvre de tuya (graffiti)». Cependant, le geste du « Roi de Kowloon » est plutôt issu de la culture lettrée traditionnelle et n'a pas de relation avec le " graffiti moderne » de l'Occident.

18. Les membres en sont Ji Xu (CTN), Dai Yan (Quaner), Song Tongshu (Yumi), Lu Jiayin (Joey), Lei Wenhao (Viga), Lu Daning (Nezha), Liu Zhen (Guantou).

19. Les membres principaux en sont 0528 , Mo, Soos et le français Als.

20. Les membres principaux en sont William Zhou, Shier, Levi, Klmi, Rtan et Sim.

21. Le fondateur de ces deux groupes est Hurricane "Hurri" Jin.

22. Pour Seth, il ne s'agit pas de graffiti. Voici son explication: «...les peintures de la rue de Kangding ne sont pas vraiment du graffiti. Le terme graffiti est spécifique au travail de lettrages et de personnages qui vient de l'école new-yorkaise qui induit l'écriture de son nom, de son groupe, la pratique du tag... (tels que Kwanyin, ABS, Mos, MCYan etc...). Même si je viens de cette école, on parle pour ce que je fais aujourd'hui (comme pour Zhang Dali par exemple) plus d'art urbain, post-graffiti, street-art ou encore art public.» - Mail de Seth à l'auteur, le 17 nov. 2015.

23. En 2003, Seth a également collaboré avec le graffeur hongkongais MC Yan que je mentionne plus haut.

24. Mail de Seth à l'auteur, le 8 déc. 2015.

25. Propos recueillis par Fan Jie (2015), « Le graffeur Shi Zheng, la ruine contient de l'espoir ». In : Journal du soir de Xinmin (Xinmin wanbao), Shanghai, le 25 janvier.

26. Traduction de Paul Demiéville (1962), Anthologie de la poésie chinoise classique, Paris, Gallimard.

27. Propos recueillis par Fan Jie, op. cit.

28. À propos de l'ensemble de la démarche de ce projet, voici l'explication précise de Seth «... suite à des peintures que j'avais faites à Xintiandi [New Heaven and Earth, il s'agit d'un ancien quartier complètement restauré et devenu aujourd'hui un des lieux le plus chic de Shanghai] en novembre précédent, je suis revenu en Chine pour peindre de nouveau dans des quartiers de Shanghai. Une amie chinoise m'a aidé à chercher des endroits pour peindre. La rue de Kandging m'a plu et j'ai décidé d'y revenir seul le lendemain. J'ai d'abord invité un artiste hongkongais britannique avec qui j'avais déjà collaboré à venir peindre là-bas avec moi "the orange blowfish". Ensuite j'ai demandé à mon amie de me présenter un calligraphe chinois pour écrire sur les murs des citations et poèmes en relation avec les personnages. Elle m'a présenté un Shi Zheng qui est venu réaliser ces calligraphies puis a peint ses propres créations. Ses amis qui l'ont accompagnée ont pris des photos, et j'imagine se sont réapproprié le projet. D'autres artistes sont venus peindre à la rue de Kangding notamment Caratoes, une street-artiste de Hong-Kong... En ce qui concerne la collaboration avec Shi Zheng elle s'est en effet bien passée malgré la barrière de la langue. Un photographe présent qui parlait anglais nous aidait à nous comprendre. » - Mail de Seth à l'auteur, le 17 nov. 2015. 
29. Commentateur du site Guangming Online (Guangming wang) (2015), «Bien traiter le graffiti consacré à la démolition, écouter avec respect la dernière chanson de la vieille maison ». In : Guangming Online. http://guancha.gmw.cn/2015-01/25/content_14631991.htm [5.11.15]

30. Propos recueillis par Louisa Lim (2011), «Hong Kong Graffiti Challenges Chinese Artist's Arrest ». In: National Public Radio, Washington, D.C. http://www.npr.org/2011/05/04/135985475/ hong-kong-graffiti-challenges-chinese-artists-arrest. [4/5/11]: «...if he can be arrested, then there's no identity we can hide behind: Being a Hong Kong citizen doesn't help anymore; being rich or social status doesn't help. There's no shield any more against this very naked power that's trying to engulf us. "

31. Journaliste anonyme (2011), «C'est de l'art ou ce sont des ordures? » In : Journal du matin Jianghuai (Jianghuaichenbao), [19.4.11]

32. Shelly Kraicer (2012), «Ai Weiwei: Never Sorry: Film review». In : Chinese Cinemas. https:// chinesecinemas.wordpress.com/2012/12/19/ai-weiwei-never-sorry-film-review/. « It was initially startling to me that many of my most liberal chinese friends (i.e., those critical of the Chinese government and system, antirepression, pro free-speech) and colleagues think very little of Ai. They consider him to be a grandstanding showman who acts out, famously raising his middle finger at the Tiananmen Gate, to court Western adulation. »

\section{ABSTRACTS}

This article treats essentially with the art of graffiti in the cities as Beijing, Shanghai, Guangzhou, Chongqing and Hong-Kong. From the history of the first graffiti artist Zhang Dali, the article develops in a chronological way by revealing the development of the graffiti undertaken by the generation born after 1980, inspired essentially by the Hip-hop culture. In these limited pages, we met the different meanings from the word tuya [graffiti] in the classical and modern periods; the large-scale marketing of the graffiti ; the graffiti as tool of the propaganda in the service of the government; The exceptional cases when, by the graffiti, emerge certain social and political sensitive subjects and the different reactions between the Hong-Kong city and the mainland china.

Cet article traite essentiellement de l'art du graffiti dans des grandes villes comme Pékin, Shanghai, Guangzhou, Chongqing et Hong Kong. A partir de la présentation du premier artiste graffeur Zhang Dali, l'article se développe d'une manière chronologique en dévoilant le développement du graffiti entrepris par la génération née après 1980, inspirée essentiellement par la culture Hip-Hop. Dans ces pages limitées, on rencontra les sens différents du mot tuya [graffiti] dans les époques classiques et modernes ; la grande ampleur de la commercialisation du graffiti; le graffiti en tant qu'outil de la propagande au service du gouvernement; les cas exceptionnels lorsque, par le graffiti, émergent certains sujets sensibles sociaux et politiques et les réactions différentes entre la ville de Hong Kong et la Chine continentale. 


\section{INDEX}

Chronological index: XXème siècle, XXIème siècle

Mots-clés: art contemporain chinois, graffiti, street art

Geographical index: Chine

\section{AUTHOR}

\section{SHIYAN LI}

Docteure en Sciences de l'Art d'Aix-Marseille Université, membre du Laboratoire d'études en Sciences des Arts (LESA), membre du Réseau Asie-Pacifique (CNRS-FMSH). Son travail de recherche porte sur la réception de la pensée extrême-orientale dans l'art occidental moderne et contemporain, la naissance de l'art contemporain chinois après la Révolution culturelle ainsi que l'étude lexicographique du vocabulaire critique de l'art chinois des XXe et XXIe siècles et ses problèmes de traduction. Elle a publié l'ouvrage Le vide dans l'art du XXème siècle : Occident/ Extrême-Orient, (Aix-en-Provence, PUP, 2014, 344p.) et de nombreux articles consacrés notamment à l'étude de l'art contemporain chinois. 